\title{
Sistemas universales de protecciones sociales como alternativa a la Cobertura Universal en Salud (CUS)
}

\author{
Universal social protection systems as an alternative to Universal \\ Health Coverage (UHC)
}

Mario Hernández ${ }^{1}$

DOI: 10.1590/0103-11042019S503

RESUMEN Los organismos financieros internacionales y de Naciones Unidas han promovido la estrategia de la Cobertura Universal en Salud (CUS) como la mejor forma de garantizar el acceso a los servicios de salud. Este artículo debate esta propuesta desde el punto de vista de su eficacia para la garantía del derecho a la salud, además de su funcionalidad para la acumulación de capital en el llamado 'complejo médico industrial y financiero’ de la salud, en el marco de la financiarización y el capitalismo cognitivo. El caso colombiano, ejemplo de la aplicación de la CUS en más de veinte años, pone en evidencia los límites de la propuesta. Como alternativa se presenta la opción de los sistemas universales de protección social, apoyado en un debate público abierto y de movilización social local y global por la defensa de la vida, no solo humana, y no reducida al acceso a servicios de salud.

PALABRAS-CLAVE Cobertura Universal de Salud. Accesibilidad a los servicios de salud. Sistemas de salud. Reforma de la atención de salud.

\begin{abstract}
The international financial organizations and United Nations have promoted the strategy of Universal Health Coverage (UHC) as the best way to guarantee access to health care services. This article discusses this proposal from the point of view of its effectiveness for the guarantee of the right to health as well as its functionality for the accumulation of capital in the so-called 'financial and industrial medical complex' of health, within the framework of financialization and cognitive capitalism. The Colombian case, an example of the application of the CUS in more than twenty years, highlights the limits of the proposal. As an alternative, the option of universal social protection systems is presented, supported by an open public debate and local and global social mobilization for the defense of life, not only human, and not reduced to access to health care services.
\end{abstract}

KEYWORDS Universal Health Coverage. Health services accessibility. Health systems. Health care reform.

1 Universidad Nacional de 


\section{Introducción}

En 1997 el Banco Interamericano de Desarrollo (BID) publicó el documento 'Pluralismo estructurado: hacia un modelo innovador para la reforma de los sistemas de salud en América Latina', del economista Juan Luis Londoño, ex ministro de salud de Colombia, y Julio Frenk, médico impulsor de la reforma del sistema de salud en México. Los autores afirmaban que los modelos de sistemas de salud existentes no podrían afrontar los incrementos en los costos de atención de una población mundial en envejecimiento. De allí su propuesta 'innovadora' de rediseño de los sistemas, con base en un arreglo institucional que permitiera una nueva relación entre los Estados y los mercados. Además de las funciones tradicionales de 'financiamiento' y 'prestación', las de 'modulación' por parte del Estado y la 'articulación' por parte de agentes de mercado, darían "el carácter 'estructurado' a este tipo de pluralismo"1(17).

En 1999, el economista Joseph Kutzin presentó la propuesta 'Hacia la cobertura universal de salud. Un marco orientado a objetivos para el análisis de políticas'2, en la reunión conjunta entre el Banco Mundial (BM) y la Organización Mundial de la Salud (OMS). Consistía en 'proteger' a los individuos del 'riesgo financiero' derivado del creciente costo de la atención médica, por medio de la 'función de seguro'. Esta propuesta sería acogida por los organismos multilaterales como Cobertura Universal en Salud (CUS), cuyo objetivo "es asegurar que todas las personas reciban los servicios sanitarios que necesitan, sin tener que sufrir penurias financieras para pagarlos"3. Este artículo pretende mostrar que, no solo la CUS no logra ni logrará el acceso de las poblaciones a servicios de salud, sino que es un discurso funcional a la acumulación de capital en el Complejo Médico Industrial y Financiero (CMIF), dentro del llamado 'capitalismo cognitivo' contemporáneo. Para sustentar este argumento se presenta, en primer lugar, las dinámicas del CMIF en el capitalismo cognitivo; en segundo lugar, la funcionalidad del fundamento neoclásico y neoinstitucional de la CUS para la acumulación de capital; en tercer lugar, la incapacidad del modelo para garantizar el derecho a la salud; y en cuarto lugar, la opción de los sistemas universales de protecciones sociales para avanzar en la garantía del derecho a la salud, más allá de la atención médica individual.

\section{El Complejo Médico Industrial y Financiero en el capitalismo cognitivo}

Se ha atribuido a Arnold Relman, editor de 'The New England Journal of Medicine' entre 1977 y 1991, el concepto de 'complejo médico industrial', por su artículo de 1980 titulado 'El nuevo complejo médico industrial'4. Pero realmente el concepto apareció en 1967, en un informe de Robb Burlage ${ }^{5}$, líder del Health Policy Advisory Center (HealthPAC) de Nueva York, en el que daba cuenta de la expansión lucrativa de los hospitales universitarios, gracias a la transferencia de recursos públicos municipales para atención subsidiada de comunidades pobres en Nueva York. Decía Burlage:

La 'industria de la salud' solía significar solo médicos y compañías farmacéuticas. Ahora comprende médicos, medicamentos, suministros hospitalarios, equipos electrónicos, computadoras, seguros médicos, construcción, bienes raíces y cadenas lucrativas de hospitales y hogares de ancianos $6(1)$.

Este proceso de corporativización de la atención médica venía gestándose desde finales del siglo XIX, cuando la medicina artesanal se transformó en científico-técnica a través de las 'mentalidades médicas'7. El 'hospital universitario', propuesto por Abraham Flexner en su informe de 1910, propició la transformación del ámbito hospitalario en fábricas de producción de servicios de salud ${ }^{\mathbf{8}}$, 
dominadas por los 'patricios' de la Asociación Médica Americana (AMA) ${ }^{9}$, mientras la industria farmacéutica materializaba el proyecto de la 'bala mágica' de Paul Ehrlich ${ }^{10}$. En los años treinta, con la aparición del seguro médico, se fueron articulando los mercados de atención, formación, investigación, innovación y medicamentos'. En 1945, el programa de Vannevar Bush, 'Ciencia, la frontera sin fin'”1, movilizó recursos públicos para innovación en salud y generó las articulaciones entre la tecnología de guerra y servicios de salud'12. Para los sesenta, el programa de subsidios a la demanda en salud para ancianos (Madicare) y familias 'pobres' (Medicaid) impulsó la configuración del 'Complejo Médico Industrial' que señalaba el Health-PAC ${ }^{13}$, asimilándolo al 'Complejo Militar Industrial' del que había hablado el presidente Eisenhower en 1961,13.

Con los pactos de Breton Woods de 1944, que crearon los organismos financieros multilaterales y el patrón dólar-oro, estas corporaciones se favorecieron. Pero la sobreacumulación de los sesenta rompió el patrón dólar-oro, primero con los 'eurodólares' que circularon por las bolsas de valores, y poco después, con la decisión de Richard Nixon de emitir dólares sin respaldo en oro en $1971^{\mathbf{1 4}}$. El libre mercado de dólares generó un ciclo inflacionario que, junto con la crisis del petróleo impulsada por la colusión entre Nixon y los países árabes entre 1973 y $1974^{15}$, y el incremento de las tasas de interés decidido por la Reserva Federal de Estados Unidos en 197914, produjeron el comienzo de la 'financiarización' de las economías, esto es, el paso del régimen industrial fordista de los Estados de bienestar keynesianos al régimen financiarizado de acumulación de corte neoliberal ${ }^{16,17}$.

Entre tanto, las grandes empresas cambiaron su financiamiento del crédito controlado según su patrimonio al esquema de expansión por la venta de acciones en el mercado abierto de valores. Esta opción les generó una progresiva dependencia de los tenedores de acciones ${ }^{\mathbf{1 8}}$. El CMI también entró en este proceso de financiarización a través de diversos mecanismos. Kelman ${ }^{\mathbf{8}}$ veía en las health manteinance organizations promovidas en la administración Nixon la incorporación decidida del sector financiero en el sistema de salud. La dinámica financiera propició procesos de monopolización como la transición de instituciones 'sin ánimo de lucro' a 'lucrativas', la conformación de consorcios entre hospitales, la compra de empresas por la industria farmacéutica para incrementar los precios, disminuir gastos en investigación e innovación y eludir impuestos ${ }^{19}$. De aquí la denominación de CMIF.

El incremento de precios en la atención médica fue también el resultado de la incorporación de la tecnología de guerra en el medio hospitalario, incluso sin suficiente discusión ni evidencia ${ }^{20}$. Estas nuevas tecnologías, como los medicamentos, estaban atadas a patentes, que en Estados Unidos aumentaron en los años cincuenta y sesenta20,21. El microprocesador basado en la pastilla de silicio (silicon chip), creado en $1971^{22}$, y el modelo binario de producción y transmisión de información, fueron creando los llamados 'bienes informacionales' en diferentes soportes y alta replicabilidad, con costos de producción decrecientes hasta cuasi cero $^{23}$, en especial en el sector financiero ${ }^{22}$. Los procesos productivos y de consumo basados en este tipo de bienes fueron dominando cada vez más la acumulación de capital en diferentes sectores, hasta atravesar la vida cotidiana de las personas. A este predominio de la acumulación basada en bienes informacionales y de conocimiento se ha denominado 'capitalismo cognitivo'24,25.

El concepto de 'propiedad intelectual' es la estrategia central en el capitalismo cognitivo. Burdeau ${ }^{26}$ denomina a este proceso 'el último cercamiento'. El copyright, como privilegio de los autores de obras culturales, y las patentes, para proteger la 'propiedad industrial' de los inventores, que venían desde el siglo XV, se transformaron a partir del Copyright Act de 1976 en Estados Unidos en Derechos de Propiedad Intelectual (DPI). Afirma Zukerfeld: 
[...] el capitalismo cognitivo se está constituyendo alrededor de las siguientes operaciones: i) la unificación de un conjunto de derechos monopólicos, ii) su legitimación bajo la expresión 'propiedad intelectual', iii) la constitución de un marco institucional que bregue por su aplicación, iv) la expansión de los derechos de propiedad intelectual en cuanto a su magnitud, duración y alcance ${ }^{27(24)}$.

La tecnología biomédica y la industria farmacéutica, protegidas por los DPI, han hecho de este sector económico uno de los más promisorios en el mundo, más aún, con la manipulación genética de bacterias con biotecnología ${ }^{28}$, en la que la industria farmacéutica fue punta de lanza, comenzando por la insulina recombinante 29 . Esto explica los exorbitantes precios de los medicamentos y los servicios médicos. En este contexto, no es extraño el cambio en el discurso técnico-político de los organismos multilaterales.

\section{Un discurso funcional a la acumulación de capital en salud}

La crisis de sobreacumulación del capitalismo en los años setenta llevó a la recurrente "solución espacio-temporal" de la que habla Harvey ${ }^{16(99)}$, facilitada por las nuevas tecnologías. Se requería ampliar los mercados y restringir la provisión directa de servicios por parte del Estado. De allí que el discurso neoliberal de la Mont Pelerin Society de Friedrich Hayek y Milton Friedman en 1947, viniera como anillo al dedo ${ }^{\mathbf{1 4}, 30}$. De hecho, el neoliberalismo implicaba un "proyecto político para restablecer las condiciones para la acumulación de capital y restaurar el poder de las élites económicas"14(25). Dentro de las fronteras de cada país, mientras se aplicaba la apertura de los mercados ${ }^{16}$, las clases propietarias ubicaron sus nichos de relacionamiento y de articulación con los nuevos flujos de acumulación ${ }^{31}$.
$\mathrm{El}$ incremento de las tasas de interés en 1979 derivó en la imposibilidad de pagar la deuda. Consciente de esta situación, el Fondo Monetario Internacional (FMI) desarrolló los 'programas de ajuste' en 1981, a cambio de la reducción del gasto social, flexibilización laboral, desregulación financiera y privatización de empresas públicas, comenzando por los fondos públicos de pensiones ${ }^{32,33}$. El primer experimento fue México en 1982, y poco después, casi todos los países de América Latina $^{32,33}$. Aunque la aplicación de la agenda neoliberal comenzó en la dictadura de Augusto Pinochet en Chile desde $1973^{\mathbf{1 4}}$, los programas de ajuste configuraron la primera agenda de reforma de los estados latinoamericanos que condujo al denominado 'Consenso de Washington'34 en 1989.

En este contexto, el $\mathrm{BM}^{35}$ presentó el informe sobre 'El financiamiento de los servicios de salud en los países en desarrollo. Una agenda para la reforma' en 1987. El fundamento del informe estaba en la diferenciación entre bienes privados y bienes públicos en salud, a partir de la teoría de la elección racional ${ }^{36}$ en economía y ciencias políticas:

Puede ser útil clasificar los bienes y servicios provistos por el sistema de salud, de acuerdo con quienes reciben los beneficios. En un extremo están los bienes netamente privados, cuyos beneficios son captados totalmente por la persona que recibe el servicio de salud, y en el otro extremo se hallan los bienes puramente públicos, cuyos beneficios perciben por igual todos los miembros de la sociedad [...] Casi siempre los consumidores están dispuestos a pagar directamente por los servicios que implican beneficios mayormente privados, pero por lo general son reacios a hacerlo por aquellos programas y servicios que benefician a la sociedad o a la comunidad como un todo35(697).

De esta posición resultaba la propuesta del BM de cobrar aranceles a los usuarios, promover los seguros médicos, atraer recursos no 
gubernamentales y descentralizar los servicios de salud ${ }^{35}$. La clave estaba en diferenciar qué le competía al mercado y qué al Estado en esta materia, en el mismo sentido en que Milton Friedman había propuesto para la educación en 195537. El informe 'Invertir en salud' de $1993^{38}$ consolidó la agenda de reforma del BM, con estrategias más refinadas: la introducción de agentes de mercado en competencia, tanto para el aseguramiento como la provisión de servicios; la concentración de los recursos públicos en acciones con 'altas externalidades'; y el subsidio a la demanda focalizado en los 'pobres' con paquetes de servicios costo-efectivos, con base en la 'carga de la enfermedad'33,39.

Varias reformas estaban en curso. La de Colombia tuvo particular interés porque incorporaba, además de la teoría de la elección racional, otros elementos de la corriente neoclásica en economía. En particular, el managed care o 'atención gerenciada', sustentado en las 'asimetrías de información' entre compradores y proveedores de atención médica, de Keneth Arrow ${ }^{\mathbf{4 0}}$, y el managed competition o 'competencia regulada' de Alain Enthoven ${ }^{\mathbf{4 1}}$. El primer modelo planteaba que la atención médica era un bien privado, pero la 'asimetría de información' entre el consumidor (el enfermo) y el proveedor (el médico) implicaría siempre una incertidumbre tal que no podría funcionar como un mercado basado en preferencias racionales ${ }^{40}$. Se requería un 'comprador inteligente' que protegiera al paciente de los desmanes del proveedor. Así se justificaba la larga experiencia de los seguros de salud en Estados Unidos y se incentivaría la 'atención gerenciada' en todos los sistemas para ampliar los mercados ${ }^{\mathbf{4 2}}$. El segundo modelo proponía regular la competencia entre aseguradores por medio del pago de un mismo valor per cápita, desde un fondo 'patrocinador', a cambio de un mismo paquete de servicios. $\mathrm{Si}$ los aseguradores querían ganancias, tendrían que prevenir los 'siniestros' ${ }^{\mathbf{4 3}}$. Estos modelos se incorporaron en Colombia con la ley $\mathrm{N}^{\mathrm{o}}$ 100 de 1993. Se creó un sistema de 'seguridad social' que incluía pensiones, riesgos laborales y salud. En el área de pensiones, se crearon los fondos privados de ahorro individual, como en Chile ${ }^{33}$, pero persistió un sistema público de prima media; en lo que hace a riesgos laborales, entraron las empresas privadas de seguros en competencia abierta; pero en salud, se hizo un ordenamiento más sofisticado, basado en los fundamentos neoclásicos. El modelo de parafiscalidad, denominado 'régimen contributivo', implicó que empleadores y trabajadores aportaran cotizaciones obligatorias. Los recursos públicos recaudados se entregaron, como una Unidad de Pago por Capitación (UPC), a cambio de un Plan Obligatorio de Salud (POS), a empresas intermediarias en competencia, denominadas Empresas Promotoras de Salud (EPS). Estas empresas debieron organizar redes de servicios para sus afiliados con instituciones prestadoras en competencia, privadas o estatales, por contrato o propias. Para el caso de las personas que demostraran ser 'pobres', a través de una encuesta domiciliaria, el Estado pagaría la UPC a empresas encargadas de un 'régimen subsidiado'. Pero esta UPC era mucho menor que la del contributivo, lo que se expresaba en el POS. En este sentido, se trataba de un modelo de seguros de salud que combinaba managed care con maneged competition, con separación entre pobres y no pobres, y un subsidio a la demanda financiado por impuestos ${ }^{\mathbf{4 4}}$.

Después de ser protagonista en la reforma colombiana, Juan Luis Londoño fue llamado al BID para reflexionar sobre su experiencia. En esa entidad se encontró con Julio Frenk, promotor sin éxito de una reforma similar en México. El resultado de su reflexión conjunta fue la propuesta del 'pluralismo estructurado'. La separación de funciones permitiría diferenciar las responsabilidades del Estado y del mercado. La 'articulación', esto es, la relación entre el fondo público que se conformara con cotizaciones o impuestos (Estado) y los prestadores en libre competencia (mercado), sería ejercida por las denominadas Organizaciones para la Articulación de Servicios de Salud (OASS) (mercado). Y estas OASS (managed care) serían 'reguladas' (managed competition) 
por el pago por UPC a cambio de un plan de beneficios, como en Colombia. De esta forma, tendrían estímulos para ejercer tres subfunciones: la administración de riesgos, la administración del acceso y la representación del consumidor. Se incorporaban así los desarrollos del discurso neoclásico en economía, fundamento de la agenda y del neoliberalismo en salud $\mathbf{4 5}$.

Un elemento más alimentó la propuesta. Se trataba del neoinstitucionalismo económico promovido por Douglas North ${ }^{\mathbf{4}}$, para quien las 'buenas instituciones' serían aquellas que lograran disminuir los costos de transacción entre los agentes, para promover el crecimiento económico y el desarrollo. El exvicepresidente del BM, William Wapenhans, en su balance sobre los fracasos de los programas de lucha contra la pobreza, incorporó los conceptos de North en la agenda del Banco ${ }^{47}$. De allí surgió un conjunto de estrategias de 'gobernanza' o 'buen gobierno', entendidas como la generación de reglas claras por parte del Estado para garantizar los derechos de propiedad y disminuir los costos de transacción. En pocas palabras, "un Estado funcional al mercado"32(60). Con base en North, Londoño y Frenk afirmaban:

[...] la discusión acerca de las opciones de reforma se centra en la necesidad de establecer reglas del juego justas y transparentes con el fin de impulsar un desarrollo armónico del sistema [...] el tipo de modulación que requiere el pluralismo no coloca obstáculos al mercado de los servicios de salud, sino que representa una condición necesaria para que este funcione de manera transparente y eficiente $\mathbf{1}^{\mathbf{1}(1,20)}$

Las reglas evitarían los efectos negativos de la descentralización, mejorarían la calidad y permitirían reorientar los recursos públicos hacia el subsidio a la demanda de los 'pobres' y a resolver altas externalidades. Se podrían así superar los excesos del Estado y las fallas del mercado, resolviendo la tensión entre “eficiencia y equidad"1(17). Poco después, el informe sobre el 'Desempeño de los sistemas de salud'48, liderado por economistas neoclásicos entre los que participaron Londoño y Frenk, otorgó el primer lugar en 'equidad financiera' y el puesto 21 total a Colombia, por encima de Cuba, Costa Rica y Brasili3,49. Con este impulso, el modelo colombiano de pluralismo estructurado se convirtió en referente mundial50.

Simultáneamente surgió la propuesta de los economistas del BM, Robert Holzmann y Steen Jørgensen ${ }^{51}$, sobre el Manejo Social del Riesgo (MSR). Según estos autores, los individuos somos 'trapecistas' que sorteamos la vida con nuestro propio esfuerzo. La adversidad impone 'riesgos financieros' que debemos asumir con ingresos o ahorros. Dado que no siempre se tienen, debería acudirse a redes de distinto tipo, para mitigar, prevenir $o$ atender los riesgos. Primero, la red familiar, que debe responder por sus miembros; segundo, la filantropía o las redes espontáneas de 'capital social'; tercero, los seguros con destinación específica; y cuarto, y en última instancia, la 'nueva protección social' organizada por el Estado, en la forma de subsidios para 'pobres' de manera temporal. Entre estos últimos estarían las Transferencias en Efectivo Condicionadas (TEC), dirigidas a las mujeres y a la inversión en su propio 'capital humano'51. El MSR ha servido para la reorganización de todas las políticas sociales. Colombia, otra vez, fue ejemplar en su aplicación, con Juan Luis Londoño de nuevo como ministro. Por la Ley 789 de 2002 se creó el 'Sistema de Protección Social' y se fusionaron los ministerios de Salud y Trabajo para crear el Ministerio de Protección Social.

No es extraño, entonces, que la propuesta de Kutzin ${ }^{2}$ se concentrara en generalizar la función de seguro de atención médica, bien fuera con seguro obligatorio, seguro subsidiado para pobres o seguro voluntario comercial. Esto permitiría proteger a las personas del riesgo financiero al tiempo que facilitaría las ganancias del CMIF ${ }^{\mathbf{9}, \mathbf{3 3}}$, en el régimen de financiarización y del capitalismo cognitivo. 


\section{La incapacidad de la CUS para garantizar el derecho a la salud}

Dado que el caso colombiano es el más ajustado a la agenda internacional de la CUS, vale la pena examinar sus resultados, de cara a la Observación General $\mathbf{1 4}$ del Comité de los Derechos Económicos, Sociales y Culturales. Este documento ratifica que "[T]odo ser humano tiene derecho al disfrute del más alto nivel posible de salud que le permita vivir dignamente"52(1). Significa que, además de la atención integral en salud,

[E]l derecho a la salud está estrechamente vinculado con el ejercicio de otros derechos humanos y depende de esos derechos [...] en particular el derecho a la alimentación, a la vivienda, al trabajo, a la educación, a la dignidad humana, a la vida, a la no discriminación, a la igualdad, a no ser sometido a torturas, a la vida privada, al acceso a la información y a la libertad de asociación, reunión y circulación ${ }^{52(2)}$.

Para la interdependencia de derechos, la agenda internacional separa la atención médica, con la CUS, por un lado, y la estrategia de 'Salud en Todas las Políticas' (SeTP), por el otro. Colombia es también el mejor ejemplo de este diseño. Para la primera, el derecho se traduce en el Plan de Beneficios en Salud (PBS); para la 'salud pública', en un Plan de Intervenciones Colectivas (PIC); y para la SeTP, se creó una 'Comisión intersectorial de salud pública'53. A pesar de la aprobación de una ley estatutaria en salud que "regula el derecho fundamental a la salud"54(1), que incluye la atención médica integral y la afectación de los 'determinantes sociales en salud', los resultados son precarios.

Como era de esperarse, Colombia reporta una cobertura de aseguramiento en salud para 2018 de $94,66 \%{ }^{55}$. Esta cobertura ha permitido incrementar el consumo de servicios de atención médica: entre 2010 y 2014, el número de 'procedimientos' en salud pasó de cerca de 190 millones a más de 250 millones $^{56}$. Esto debe alegrar a los dueños de los grandes negocios del CMIF. Sin embargo, este aumento en el uso de servicios no implica un goce efectivo del derecho a la atención en salud. Por el contrario, produce o reproduce inequidades acumuladas.

Entre 1997 y 2012, según la Encuesta Nacional de Calidad de Vida (ENCV), el acceso a servicios entre quienes afirmaron haber necesitado atención en el último mes, disminuyó de $79,1 \%$ a $75,5 \%$, con grandes diferencias regionales ${ }^{57}$. Si bien dentro de las razones para no acceder disminuyó la 'falta de dinero', de 42,3\% a $11,5 \%$, gracias al aseguramiento, la consideración de 'mal servicio o cita distanciada en el tiempo' pasó de 1,4\% a 8,3\%, la de 'el centro de atención queda lejos' se incrementó de $4,2 \%$ a 5,8\%, y 'muchos trámites para la cita' de 3,4\% a $5,8 \%$, todas ellas relacionadas con "barreras de acceso"57(19). Estas barreras pueden ser geográficas, administrativas, culturales y de infraestructura, como lo muestran estudios basados en la percepción de los usuarios ${ }^{58}$ y de los profesionales y trabajadores de la salud ${ }^{59}$. Abadía y Oviedo ${ }^{60}$ caracterizaron esta situación como 'itinerarios burocráticos' que terminan en sufrimiento, complicaciones, discapacidad y muertes prevenibles. De hecho, entre 1998 y 2011 se reportaron 2.677 .170 muertes, de las cuales 1.427 .535 (53\%) fueron clasificadas como "causas evitables"61(5). Las 'acciones de tutela' por la negación de servicios de salud alcanzó en 2018 la cifra de 207.734, con notorias desigualdades regionales ${ }^{62}$. En 2014, se encontró que cerca del $70 \%$ de las tutelas fueron por servicios incluidos en el $\mathrm{PBS}^{63}$.

Los servicios de mayor complejidad y el personal de salud se concentran en seis ciudades, mientras se ha perdido la capacidad resolutiva del primero y el segundo nivel, y los incentivos apuntan al "número de actividades realizadas, mas no en la búsqueda de los resultados en salud"64(18).

La separación estructural por regímenes ha significado desigualdades inaceptables. 
Por ejemplo, entre 2011 y $2014,61,26 \%$ de las atenciones en salud registradas en el sistema fueron de afiliados al régimen contributivo, frente a $32,05 \%$ del subsidiado ${ }^{56}$. En Bogotá se encontró que las gestantes del régimen subsidiado tenían, de manera sostenida entre 2005 y 2011, el doble de riesgo de morir por causas relacionadas con el embarazo que las del régimen contributivo ${ }^{65}$. Entre $2011 \mathrm{y}$ 2014 se verificó que la población del régimen subsidiado presentaba $27 \%$ a más de tasa de mortalidad general, $28 \%$ a más de tasa de mortalidad perinatal, $64 \%$ a más de razón de mortalidad materna general y $35 \%$ a más de tasa de mortalidad de menores de 5 años que la población afiliada al contributivo ${ }^{56}$.

Con base en la comparación de las encuestas de calidad de vida de 2008 y 2015 , se encontró que los campesinos, obreros agropecuarios y trabajadores domésticos se hallan en peor condición de salud de manera consistente, respecto de los trabajadores por cuenta propia, y mucho peor que los empleados, profesionales y técnicos, y directivos ${ }^{67}$. Los indicadores de morbimortalidad son varias veces peores en la población indígena y afrocolombiana que en la mestiza o sin etnia reconocida ${ }^{67,68}$. Por ejemplo, la razón de mortalidad materna en la población indígena fue 6,8 veces mayor y la de mujeres afrocolombianas, dos veces mayor que el promedio nacional. La tasa de mortalidad infantil fue 2,6 veces mayor entre los indígenas y 1,3 veces mayor en los afrocolombianos que la media nacional ${ }^{64}$. Se ha demostrado que, con la refamiliarización del cuidado de los enfermos, se incrementó la carga de las mujeres en los hogares ${ }^{69}$.

En 2012 se encontró que "algunas de las regiones con mayores necesidades de servicios médicos fueron las que contaban con menores prestadores por habitante"57(10). La poca rentabilidad del aseguramiento en áreas geográficas con menor densidad poblacional, pero con mayores necesidades en salud y mayores impactos del conflicto armado, ha generado menor acceso a servicios y peores resultados en salud en la zona rural, a pesar de haberse ofrecido una UPC diferencial por población dispersa ${ }^{64}$.

Existen deudas acumuladas entre el fondo público y las EPS, y entre estas y los prestadores públicos y privados, aún sin cálculo definitivo y sin resolver ${ }^{70}$. El control del gasto por parte de las EPS se traslada a los prestadores por distintas vías, como pagos por capitación de alto riesgo, tarifas leoninas y 'glosas de facturas $^{\mathbf{7 1}}$, lo que genera crisis hospitalaria ${ }^{\mathbf{7 2 , 7 3}} \mathrm{y}$ precarización laboral progresiva ${ }^{74}$, mediante despidos y tercerización ${ }^{75}$. Mientras tanto, se afecta la autonomía profesional ${ }^{76}$ y los médicos generales han entrado en proletarización ${ }^{77}$. La privatización de la prestación de servicios alcanzaba el 97,54\% en 2014 y cerca de $57 \%$ de los prestadores se ubicaban en las ciudades y departamentos más ricos ${ }^{64}$. Desde el punto de la salud pública, la asimilación con el PIC, la pérdida de capacidad técnica y recursos, en especial de los municipios más pequeños, ha generado un debilitamiento de la 'rectoría' y de las políticas y programas, así como la falta de liderazgo para la intersectorialidad o la SeTP64. Esto no es extraño, porque el aseguramiento individualiza y desterritorializa.

El Ministerio afirma:

Se puede concluir que el supuesto inicial de mercados de servicios modulados por el mercado no ha posibilitado una regulación extensiva de acuerdo con el interés del usuario y la consolidación del derecho a la salud de toda la población [...] Este efecto es consecuencia del propio diseño de un modelo de seguridad social, donde se impulsa un enfoque de prestación orientado hacia la cobertura de las contingencias financieras para las familias derivadas del costo de la enfermedad $\mathbf{6 4}(\mathbf{1 7 , 1 8 )}$.

A pesar del intento del Ministerio por 'alinear los incentivos' de los agentes a partir de su Política de Atención Integral en Salud (PAIS) ${ }^{78}$, los resultados todavía no se han visto. Acudir a la elección racional de los agentes profundiza el individualismo y no permite construir salud. El asegurador ve en cada 
afiliado una UPC que debe tratar de no gastar. Este control del gasto, del cual se deriva la ganancia, explica el conjunto de problemas descritos. Entre tanto, la 'modulación', definida desde el centro, ha debilitado al Estado en los territorios, de manera que, por esta vía, no es posible garantizar el goce efectivo del derecho a la salud, en todas las dimensiones que plantea la Observación General 14 de 2000.

\section{Sistemas universales de protecciones sociales como opción teórico-política}

Si la CUS es funcional a la acumulación de capital en el CMIF y reproduce la desigualdad social, la opción teórico-política debe ser un nuevo arreglo institucional capaz de contribuir a la construcción social de salud con alcance global. Los sistemas de seguridad social y de salud europeos son el resultado de un proceso histórico específico que no es posible repetir ${ }^{79,80}$. En América Latina nunca se realizó el Estado de bienestar, debido a trayectorias sociopolíticas particulares que deben ser reconocidas ${ }^{\mathbf{8 1}, 82}$. Se trata de construir colectivamente sistemas universales de protecciones sociales atados a la condición de seres humanos interdependientes, con la misma dignidad, como valor no transable. Pero es necesario ir un paso más allá, para avanzar en la conciencia de la interdependencia con el orden natural planetario y universal. Esto quiere decir que no se trataría de sistemas dedicados solo a la salud de las personas, sino al cuidado de la vida, con perspectiva de género y en clave de 'derechos de la naturaleza'. Esta ruta permitiría asumir con todo rigor propuestas como la del 'Buen Vivir/Vivir Bien'83 de nuestros pueblos ancestrales americanos y de otros continentes. En ellos se plantea la alternativa 'al' desarrollo, no alternativas 'de' desarrollo, porque parten de una relación ser humano-naturaleza de interdependencia y no de aprovechamiento de la naturaleza como recurso para satisfacción humana. Esto implica un enorme reto para las sociedades actuales en términos de justicia ambiental, en diálogo con propuestas como la de 'los bienes comunes' ${ }^{84}$, el 'decrecimiento' 85 , la agroecología y la soberanía alimentaria ${ }^{86}$.

Esta apuesta implica superar la lógica individualista con una propuesta solidaria en la que cada cual aporta según su capacidad y recibe según su necesidad, en sentido colectivo. Esta posición solidaria y colectivista tiene como consecuencia que los sistemas universales de protecciones sociales deben apoyarse en esquemas progresistas de fiscalidad, basados en criterios explícitos de justicia tributaria.

Los diseños institucionales deben ser sistemas situados, capaces de dar respuesta a la diversidad socioterritorial y cultural, de manera que, a partir de referentes generales se realicen adaptaciones a las necesidades locales, desde un verdadero diálogo de saberes en cada territorio. Este enfoque descentralizado y de coconstrucción democrática requiere repensar el asunto del territorio y del conocimiento. En el primer aspecto, es necesario acudir al acumulado de la corriente de pensamiento de la geografía crítica latinoamericana ${ }^{87}$. Desde esta perspectiva se entiende el territorio como un producto social y como un productor de sociedad; es el resultado de un proceso de 'territorialización', que resulta de la dinámica entre 'territorialidades' en conflicto, en relaciones de poder que expresan en lo local la conflictividad global ${ }^{88}$. Comprender los territorios permite construir colectivamente estrategias de superación de los conflictos socioambientales, desde una justicia ambiental democrática y protectora de la vida. Esta perspectiva dialoga profundamente con la corriente latinoamericana de la medicina social y la salud colectiva, en la medida en que se comprenden los procesos de deterioro o de protección de la vida de manera socioterritorial e histórica, desde la categoría intermedia de 'modo de vida' y del metabolismo sociedad-naturaleza ${ }^{89}$.

Por otra parte, si el núcleo duro del capitalismo cognitivo está en los DPI, se impone 
construir un ordenamiento mundial que permita materializar la idea y el valor del conocimiento como un bien común, producto social, de generación colectiva y acceso abierto ${ }^{90}$. Esto implicaría contar con fondos públicos nacionales y transnacionales para la investigación y la innovación colectiva, de manera que los productos del trabajo científico-técnico puedan ser de acceso libre, rompiendo con el concepto de 'propiedad intelectual'. Otro tipo de reconocimiento de los investigadores, creadores, innovadores e inventores tendría que surgir de pactos internacionales.

El arreglo institucional específico en materia de servicios de salud tendría que partir de un esquema de financiamiento público, a partir de sistemas tributarios progresivos. La administración de estos recursos debería hacerse por instituciones públicas, territorializadas, según territorios sociales más que por límites político-administrativos. Las decisiones sobre la orientación de los recursos en cada territorio serían adoptadas por cuerpos colegiados democráticos. La organización de redes de servicios debe tener un carácter transcultural, adecuado a necesidades comunitarias y territoriales, con instituciones de naturaleza múltiple, pero no lucrativas. Desde este tipo de institucionalidad es posible proponer políticas de personal, formación, ciencia, tecnología e innovación que respondan a las necesidades, más que a modelos neocoloniales. Esta conciencia de territorio permite la articulación entre sectores para el cuidado de la vida, humana y no humana.

Pero no basta proponer un nuevo arreglo institucional. Los sistemas de salud y de protección social son el producto de la correlación de fuerzas en cada sociedad, en medio del capitalismo global. Se requiere un debate profundo, desde la movilización de la sociedad y la confluencia de diferentes sectores sociales, políticos y económicos alrededor de una nueva propuesta, legítima y colectivamente construida, con la potencia suficiente como para afrontar el poder real del CMIF. Las sociedades transforman sus realidades. Polanyi ${ }^{91}$ mostró que las sociedades europeas del siglo XX pusieron límites al mercado autorregulador del siglo XIX. Que sean las sociedades del siglo XXI las que superen la recomposición neoliberal de los mercados, para proteger la vida humana y la del planeta.

\section{Colaborador}

Hernández M (0000-0002-3996-7337)* é responsável pela elaboração do manuscrito. 


\section{Referencias}

1. Londoño JL, Frenk J. Pluralismo estructurado: hacia un modelo innovador para la reforma de los sistemas de salud en América Latina. Documento de Trabajo 353. Washington, DC: Banco Interamericano de Desarrollo; 1997.

2. Kutzin J. Towards Universal Health Care Coverage. A Goal-oriented Framework for Policy Analysis. Washington (DC): The World Bank; 2000.

3. Organización Mundial de la Salud. ¿Qué es la cobertura sanitaria universal? [internet] Ginebra: Organización Mundial de la Salud; [fecha desconocida]. [acceso en 2019 oct 6]. Disponible en: https://www. who.int/universal_health_coverage/es/.

4. Relman AS. The new medical-industrial complex. N. Eng. J. Med. 1980; 303(17):963-970.

5. Burlage RK. The Municipal Hospital Affiliation Plan in New York City: a Case Study and Critique. Milbank Mem. Fund Q. 1968; 46(1):171-201.

6. Burlage RK. Editorial. The Medical Industrial Complex. Health-PAC. 1969 (Nov): 1-12.

7. Laín EP. Historia de la Medicina. Madrid: Salvat; 1978.

8. Kelman S. Toward the Political Economy of Medical Care. Inquiry. 1971; 8(3):30-38.

9. Starr P. La transformación social de la medicina en los Estados Unidos de América. México: Secretaría de Salud, Fondo de Cultura Económica; 1991.

10. Goodman J. Pharmaceutical Industry. En: Cooter $\mathrm{R}$, Pickstone J, editores. Companion to Medicine in the Twentieth Century. Abingdon: Routledge; 2003. p. 141-154.

11. Bush V. Ciencia, la frontera sin fin. Un informe al presidente, julio de 1945. Redes. 1999; 7(14):89-137.

12. Blume S. Medicine, Technology and Industry. En: Cooter R, Pickstone J, editores. Companion to Me- dicine in the Twentieth Century. Abingdon: Routledge; 2003. p. 171-185.

13. Ehrenreich B, Ehrenreich J. American Health Empire. Power, Profits, and Politics. A Report from the Health Policy Advisor Center (Health-PAC). New York: Random House; 1971.

14. Harvey D. Breve historia del neoliberalismo. Madrid: Akal; 2007.

15. Harvey D. El nuevo Imperialismo. Madrid: Akal; 2007.

16. Jessop R. El futuro del Estado capitalista. Madrid: Catarata; 2008.

17. Giraldo C. Financiarización: un nuevo orden social y político. En: Salama P, Marques-Pereira J, Lautier B, et al. Sistemas de protección social: entre la volatilidad económica y la vulnerabilidad social. Bogotá: Universidad Nacional de Colombia, Facultad de Ciencias Económicas, Centro de Investigaciones para el Desarrollo-CID; 2005. p. 233-283.

18. Chesnais F, Plihon D. Las trampas de las finanzas mundiales. Madrid: Akal; 2003.

19. Burlage R, Anderson M. The Transformation of the Medical-Industrial Complex: Financialization, the Corporate Sector, and Monopoly Capital. En: Waitzkin H, editor. Health Care under the knife. Moving Beyond Capitalism for Our Health. New York: Monthly Review Press; 2018. p. 69-82.

20. Waitzkin H. El Mercado internacional de productos y servicios de salud. In: Waitzkin H. Medicina y salud pública al final del imperio. Bogotá: Universidad Nacional de Colombia, Facultad de Medicina, Centro de Historia de la Medicina; 2013. p. 57-80.

21. Silverman M, Lee PR. Píldoras, ganancias y política. México (DF): Siglo XXI; 1983.

22. Pérez C. Revoluciones tecnológicas y capital financiero. La dinámica de las grandes burbujas financie- 
ras y las épocas de bonanza. México: Siglo XXI; 2004.

23. Zukerfeld M. Capitalismo cognitivo, trabajo informacional y un poco de música. Nómadas. 2008; (55):5265.

24. Rodríguez López E, Sánchez Cedillo R. Capitalismo cognitivo, propiedad intelectual y creación colectiva. Madrid: Traficantes de Sueños; 2004.

25. Míguez P. Del General Intellect a las tesis del "capitalismo cognitivo": aportes para el estudio del capitalismo del siglo XXI. Bajo el Volcán, 2013; 13(21):27-57.

26. Bordeau I. The Last Great Enclosure: The Crisis of the General Intellect. WorkingUSA, J. Lab. Soc. 2015; (18):649-663.

27. Zukerfeld M. El rol de la propiedad intelectual en la transición hacia el capitalismo cognitivo. Argumentos. [internet]. 2008 [acceso en: 2019 oct 16]; (9):1-25. Disponible en: http://biblioteca.clacso.edu.ar/Argentina/iigg-uba/20120626041627/9_3.pdf.

28. Cooper M. Life as surplus: biotechnology and capitalism in the neoliberal era. Washington, DC: University of Washington Press; 2008.

29. Skyler JS. Human Insulin of Recombinant DNA Origin: Clinical Potential. Diabetes care. 1982; 5(sup2): 181-186.

30. Anderson P. El despliegue del neoliberalismo y sus lecciones para la izquierda. In: Vega Cantor R, editor. Marx y el Siglo XXI. Una defensa de la Historia y del Socialismo. Santafé de Bogotá: Pensamiento Crítico; 1997. p. 353-369.

31. Katz C. Bajo el imperio del capital. Bogotá: Espacio Crítico; 2011.

32. Burgos G. Estado de derecho y globalización. El papel del Banco Mundial y las reformas institucionales en América Latina. Bogotá: Universidad Nacional de Colombia, Facultad de Derecho, Ciencias Políticas y Sociales; 2009.
33. Armada F, Muntaner C, Navarro V. Neoliberalism and Health and Social Policy. Health and social security reforms in Latin America: the convergence of the World Health Organization, the World Bank, and Transnational Corporations. Int. J. Health Ser. 2001; 31(4):729-768.

34. Williamson J. What Washington Means by Policy Reform. En: Latin American Adjustment: how much has happened. [internet] Washington, DC: Institute for International Economics; 1990. [acceso en 2019 oct 19]. Disponible en: https://web.archive.org/ web/20090625085003/http://www.iie.com/publications/papers/paper.cfm?ResearchID $=486$.

35. Banco Mundial, Departamento de Población, Salud y Nutrición. El financiamiento de los servicios de salud en los países en desarrollo. B. Of. San. Panam. 1987; 103(6):695-709.

36. Amadae SM. Rationalizing Capitalist Democracy. The Cold War and the Origins of Rational Choice Liberalism. Chicago: The University of Chicago Press; 2003.

37. Friedman M. The Role of Government in Education. [internet]. En: Solo RA, editor. Economics and the Public Interest. New Jersey: Rutgers University Press; 1955. [acceso en 2019 oct 10]. Disponible en: https://la.utexas.edu/users/hcleaver/330T/350kPE EFriedmanRoleOfGovttable.pdf .

38. Banco Mundial. Invertir en salud. Informe sobre el desarrollo mundial 1993. Washington (DC): Banco Mundial; 1993.

39. Laurell AC, López-Arellano O. Market commodities and poor relief: The World Bank proposal for health. In: Navarro V, editor. The Political Economy of Social Inequalities. Consequences for Health and Quality of Life. New York: Baywood; 2002. p. 191-208.

40. Arrow K. Uncertainty and the Welfare Economics of Medical Care. Am. Econ. Review. 1963; 53(5):941-973.

41. Enthoven A. Managed Competition: an agenda for action. Health Aff. 1988; 7(3):25-47. 
42. Iriart C, Mehry EE, Waitzkin H. La atención gerenciada en América Latina. Transnacionalización del sector salud en el contexto de la reforma. Cad. Saúde Pública. 2000; 16(1):95-105.

43. Enthoven AC, Kronich R. Universal Health Insurance through Incentives Reform. JAMA. 1991; (265):25322536.

44. Hernández M. El debate sobre la Ley 100 de 1993: antes, durante y después. En: Franco, S, editor. La Salud Pública hoy. Enfoques y dilemas contemporáneos en Salud Pública. Bogotá: Universidad Nacional de Colombia, Facultad de Odontología; 2003. p. 463-479.

45. Hernández M. Neoliberalismo en salud: desarrollos, supuestos y alternativas. En: Restrepo DI, editor. La falacia neoliberal. Crítica y alternativas. Bogotá: Universidad Nacional de Colombia; 2003. p. 347-361.

46. North D. Instituciones, cambio institucional y desempeño económico. México, DF: Fondo de Cultura Económica; 1993.

47. Wapenhans W. Effective Implementation: key to Development Impact. Washington, DC: The World Bank, Portafolio Management Task Force; 1992.

48. Organización Mundial de la Salud. Informe sobre la salud en el mundo 2000. Mejorar el desempeño de los sistemas de salud. Ginebra: OMS; 2000.

49. Hernández M. El enfoque sociopolítico para el análisis de las reformas sanitarias en América Latina. Rev. Fac. Nac. Salud Pública. 2001; 19(1):57-70.

50. Londoño JL. Managing Competition in the Tropics: Health Care Reform in Colombia. En: Molina CG, Núñez del Arco J, editores. Health Services in Latin America and Asia. Washington, DC: Inter-American Development Bank; 2001. p. 136-165.

51. Holzmann R, Jørgensen S. Social Risk Management: A New Conceptual Framework for Social Protection and Beyond. Social Protection Discussion Paper Series $\mathrm{N}^{\mathrm{o}}$ 6. Washington, DC: The World Bank, Social Protection Unit, Human Development Network; 2000.
52. Naciones Unidas, Consejo Económico y Social, Comité de Derechos Económicos, Sociales y Culturales. El derecho al disfrute del más alto nivel posible de salud. 11/08/2000. E/C.12/2000/4, CESCR Observación General 14. [internet] Ginebra: Naciones Unidas, Consejo Económico y Social; 2000. [acceso en 2019 oct 20]. Disponible en: https://www.acnur. org/fileadmin/Documentos/BDL/2001/1451.pdf.

53. Colombia. Ministerio de Salud y Protección Social. Comisión intersectorial de salud pública. [internet] Bogotá (DC): Ministerio de Salud y Protección Social. [fecha desconocida]. [acceso en 2019 oct 20]. Disponible en: https://www.minsalud.gov.co/salud/publica/epidemiologia/Paginas/comision-intersectorial-de-salud-publica.aspx.

54. Colombia. Ley $\mathrm{N}^{\circ} 1751$, de 16 de febrero de 2015. Por medio de la cual se regula el derecho fundamental a la salud y se dictan otras disposiciones. Diario Oficial No 49.427. 16 de febrero de 2015.

55. Colombia. Ministerio de Salud y Protección Social. Comportamiento del aseguramiento. [internet] Bogotá, DC: Ministerio de Salud y Protección Social. [fecha desconocida]. [acceso en: 2019 octubre 20]. Disponible en: https://www.minsalud.gov.co/proteccionsocial/Regimensubsidiado/Paginas/coberturas-del-regimen-subsidiado.aspx.

56. Asociación Colombiana de Empresas de Medicina Integral (ACEMI). Cifras e indicadores del sistema de salud. [internet]. Bogotá, DC: ACEMI; 2017. [acceso en 2019 oct 20] Disponible en: https://issuu.com/ acemi/docs/informe_cifras_2017.

57. Ayala García J. La salud en Colombia: Más cobertura pero menos acceso. Documentos de Trabajo sobre Economía Regional. Cartagena: Banco de la República, Centro de Estudios Económicos Regionales; 2014.

58. Restrepo-Zea JH, Silva-Maya C, Andrade-Rivas F, et al. Acceso a servicios de salud: análisis de barreras y estrategias en el caso de Medellín, Colombia. Rev. Gerenc. Polít. Salud. 2014; 13(27): 242-265.

59. Vargas J, Molina G. Acceso a los servicios de salud en 
seis ciudades de Colombia: limitaciones y consecuencias. Rev. Fac. Nac. Salud Pública. 2009; 27(2):121-130.

60. Abadía C, Oviedo D. Bureaucratic Itineraries in Colombia. A theoretical and methodological tool to assess managed-care health care system. Soc. Sci. Med. 2009; (68):1163-1160.

61. Colombia. Instituto Nacional de Salud, Observatorio Nacional de Salud. Tercer Informe. ONS: Mortalidad evitable en Colombia para 1998-2011. Resumen ejecutivo. Bogotá, DC: Imprenta Nacional de Colombia; 2014.

62. Colombia. Defensoría del Pueblo. La tutela y los derechos a la salud y a la seguridad social, 2018. Bogotá, DC: Defensoría del Pueblo; 2019.

63. Colombia. Defensoría del Pueblo. La tutela y los derechos a la salud y a la seguridad social, 2014. Bogotá, DC: Defensoría del Pueblo; 2015.

64. Colombia. Ministerio de Salud y Protección Social. Política de Atención Integral en Salud. Un sistema de salud al servicio de la gente. [internet]. Bogotá, DC: MSPS; 2016. [acceso en 2019 octubre 20]. Disponible en: https://www.minsalud.gov.co/sites/rid/Lists/BibliotecaDigital/RIDE/DE/modelo-pais-2016.pdf.

65. Martínez F, Hernández M. Mortalidad materna e infantil en Bogotá. Seguimiento y análisis de inequidades 2005-2011. En: Restrepo D, Hernández M, editores. Inequidad en salud en Bogotá. Convocatoria para la acción colectiva. Colección La seguridad social en la encrucijada, Tomo 7. Bogotá, DC: Secretaría Distrital de Salud de Bogotá; Universidad Nacional de Colombia, Facultad de Ciencias Económicas, Centro de Investigaciones para el Desarrollo-CID; 2012. p. 285-328.

66. Colombia. Instituto Nacional de Salud, Observatorio Nacional de Salud. Clase Social y Salud; Octavo Informe Técnico. Bogotá, DC: Imprenta Nacional; 2016.

67. Colombia. Instituto Nacional de Salud, Observatorio Nacional de Salud. Informe Nacional de las Desigualdades Sociales en Salud en Colombia. Bogotá, DC: Imprenta Nacional de Colombia; 2015.
68. Fresneda O, Martínez F. Inequidades en el derecho a la vida: análisis de la mortalidad por causas evitables en Bogotá (1998-2004). En: Restrepo DI, editor. Equidad y salud. Debates para la acción. Colección La seguridad social en la encrucijada, Tomo 4. Bogotá: Secretaría Distrital de Salud de Bogotá, Universidad Nacional de Colombia, Facultad de Ciencias Económicas, Centro de Investigaciones para el Desarrollo-CID; 2007. p. 247-295.

69. Hernández Bello A. Política sanitaria y cuidado de la salud en los hogares en Colombia: acumulación e inequidad de género. [tesis]. Bogotá, DC: Universidad Nacional de Colombia; 2015. 322 p.

70. Montes S. "Deuda del sistema de salud se acercaría a \$17 billones”: Fabio Aristizábal. [internet] La República [2019 junio 1]. [acceso en 2019 oct 21] Disponible en: https://www.larepublica.co/economia/ deuda-del-sistema-de-salud-se-acercaria-a-17-billones-fabio-aristizabal-2869182.

71. Martínez F. La crisis de la seguridad social en salud

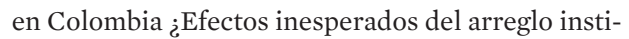
tucional? [internet] Bogotá, DC: Fundación para la Investigación y Desarrollo de la Salud y la Seguridad Social FEDESALUD; 2013. [acceso en 2019 oct 20] Disponible en: http://www.fedesalud.org/documentos/EstudiosFD_15_Crisis-seguridad-social-salud-Colombia.pdf.

72. Asociación Colombiana de Empresas Sociales del Estado y Hospitales Públicos. ACESI califica actual situación como insostenible para los hospitales. [internet] Bogotá: ACESI; 2015. [acceso en 2019 oct 20] Disponible en: http://acesi.com.co/?p=713 .

73. Asociación Colombiana de Hospitales y Clínicas. $38^{\circ}$ Informe de seguimiento de cartera hospitalaria con corte a junio 30 de 2017. [internet] Alcalá: Hospital San Vicente de Paul; 2018. [acceso en: 2019 oct 20]. Disponible en: https://hospital-alcala.micolombiadigital.gov.co/sites/hospital-alcala/content/files/000005/227_ultimo-informe-cartera-a-junio-de-2017-con-y-sin-deterioros-2410-2017.pdf.

74. Asalariados denuncian precarización. Sector salud re- 
quiere de un estatuto laboral [internet] El Nuevo Siglo [2018 mayo 11]. [acceso en 2019 oct 20]. Disponible en: https://www.elnuevosiglo.com.co/node/177125.

75. Escuela Nacional Sindical, Agencia de Información Laboral. "La tercerización laboral en la salud, se paga con vidas”, presidente de Anthoc. [internet]. Medellín: Escuela Nacional Sindical; 2017. [acceso en 2019 oct 20]. Disponible en: http://ail.ens.org.co/mundo-sindical/tercerizacion-en-salud-se-paga-con-vidas/.

76. ¿Hay respeto por la autonomía médica en Colombia? [internet] Semana [2019 mayo 26]. [acceso en 2019 octubre 20]. Disponible en: https://www.semana.com/ nacion/articulo/foro-autonomia-medica/617093.

77. Ardila A. Neoliberalismo y trabajo médico en el Sistema General de Seguridad Social en Salud. De la profesión liberal al trabajo explotado. [tesis]. Bogotá, DC: Universidad Nacional de Colombia; 2016. 141 p.

78. Colombia. Ministerio de Salud y Protección Social. Resolución $\mathrm{N}^{\circ} 429$, de 17 de febrero de 2016. Por medio de la cual se adopta la Política de Atención Integral en Salud. Diario Oficial No 49.794. 22 de feb 2016.

79. Esping-Andersen G. The Three Worlds of Welfare Capitalism. Princeton: Princeton University Press; 1990.

80. Heidenheimer AJ. The Development of Welfare States in Europe and America. New Brunswick: Transactions; 2009.

81. Fleury S. Estado sin ciudadanos. Seguridad social en América Latina. Buenos Aires: Lugar Editorial; 1997.

82. Hernández M. La fragmentación de la salud en Colombia y Argentina. Una comparación sociopolítica. 1880-1950. Bogotá, DC: Universidad Nacional de Colombia; 2004.

83. Hidalgo Flor F, Márquez Fernández A. Contrahegemonía y Bien Vivir. Quito: Universidad Central del Ecuador; Universidad del Zulia - Venezuela; 2012.
84. Zibechi R, Hardt M. Preservar y compartir: Bienes comunes y movimientos sociales. Buenos Aires: Mardulce; 2013.

85. Ecologistas en Acción. El decrecimiento, camino hacia la sostenibilidad. [internet]. Madrid: Ecologistas en Acción; 2007. [acceso en 2019 oct 21]. Disponible en: https://www.ecologistasenaccion.org/13381/el-decrecimiento-camino-hacia-la-sostenibilidad/.

86. Vía Campesina. Las luchas de la Vía Campesina por la reforma agraria, la defensa de la vida, la tierra y los territorios. [internet] [fecha desconocida] [acceso en 2019 oct 21]. Disponible en: https://viacampesina.org/es/wp-content/uploads/sites/3/2017/10/ Publication-of-Agrarian-Reform-ES.compressed.pdf.

87. Urquijo PS, Bocco V. Pensamiento geográfico en América Latina: retrospectiva y balances generales. Investigaciones Geográficas, Boletín. 2016; (90):155-175.

88. Betancourt M, Hurtado LM, Porto-Gonçalves CW. Tensiones territoriales y políticas públicas de desarrollo en la Amazonia. Buenos Aires: Consejo Latinoamericano de Ciencias Sociales - CLACSO; 2015.

89. Borde E, Torres-Tovar M. El territorio como categoría fundamental para el campo de la salud pública. Saúde debate. 2017; 41(esp): 264-275.

90. Reygadas L. La biblioteca de Babel: dilemas del conocimiento como bien común en América Latina. [internet] Buenos Aires: CLACSO; [2014] [acceso en 2019 oct 21]. Disponible en: http://biblioteca.clacso. edu.ar/clacso/becas/20141124073126/LabibliotecadeBabelDilemasdelconocimientocomobiencomun. pdf.

91. Polanyi K. La gran transformación. Critica del liberalismo económico. Madrid: La Piqueta; 1997.

Recibido en 25/10/2019

Aprobado en 11/04/2019

Conflicto de intereses: inexistente

Apoyo financiero: no hubo 